\title{
Intraoperatively measured tumor size and frozen section results should be considered jointly to predict the final pathology for lung adenocarcinoma
}

\author{
Erjia Zhu ${ }^{1} \cdot$ Huikang $\mathrm{Xie}^{2} \cdot$ Chenyang $\mathrm{Dai}^{1} \cdot$ Liping Zhang $^{2} \cdot$ Yan Huang $^{2} \cdot$ Zhengwei Dong $^{2} \cdot$ Junhong Guo $^{2} \cdot$ \\ Hang Su${ }^{1} \cdot$ Yijiu Ren ${ }^{1} \cdot$ Pingfan $\mathrm{Shi}^{3} \cdot$ Ranran $\mathrm{Fu}^{3} \cdot$ Shuang $\mathrm{Qin}^{3} \cdot$ Chunyan $\mathrm{Wu}^{2} \cdot$ Chang Chen ${ }^{1}$
}

Received: 26 September 2017 / Revised: 28 February 2018 / Accepted: 3 March 2018 / Published online: 11 May 2018

(c) United States \& Canadian Academy of Pathology 2018

\begin{abstract}
Invasive adenocarcinoma intraoperatively misdiagnosed as adenocarcinoma in situ or minimally invasive adenocarcinoma is more likely to undergo potentially insufficient resection. The purpose of our study was to evaluate the diagnostic accuracy of frozen section. We retrospectively reviewed 1,111 lung adenocarcinomas from January to March 2016 to evaluate the diagnostic performance of frozen section. A derivation cohort consisting of 436 cases of adenocarcinoma in situ or minimally invasive adenocarcinoma diagnosed by frozen section in the same period were analyzed to find predictive factors for invasive adenocarcinoma as the final diagnosis. Validation cohorts (first: April to June 2016, second: January to March 2015) were included to confirm the results. The overall concordance rate between frozen section and final diagnosis was 92\%. Most frozen section errors were underestimation. The sensitivity of frozen section diagnosis for minimally invasive adenocarcinoma (74\%) was significantly lower than others. Intraoperatively measured tumor size was the only independent factor for invasive adenocarcinoma as the final diagnosis $(<1 \mathrm{~cm}$ : $2 \%$, reference; $1-1.4 \mathrm{~cm}$ : $15 \%$, odds ratio, $5.678 ;>1.5 \mathrm{~cm}$ : $18 \%$, odds ratio, $5.878 ; P=0.001)$ in the derivation cohort, and was confirmed by validation cohorts. Fifty-nine misdiagnosed invasive adenocarcinomas in the three cohorts consisted of 54 lepidic predominant type, 1 papillary and 4 acinar predominant type. There were no positive N1, N2 node, pleural, lymphatic and vascular invasion cases found. Thirtyseven $(37 / 59,63 \%)$ cases of misdiagnosis were attributed to sampling error, which was the main reason. Our study suggests that adenocarcinoma in situ or minimally invasive adenocarcinoma $\geq 1 \mathrm{~cm}$ by frozen section were more likely to be invasive adenocarcinoma because of sampling error. Frozen section diagnosis of adenocarcinoma in situ or minimally invasive adenocarcinoma should be considered cautiously for tumors $\geq 1 \mathrm{~cm}$ to avoid potentially insufficient resection.
\end{abstract}

These authors contributed equally: Erjia Zhu, Huikang Xie, Chenyang Dai.

Electronic supplementary material The online version of this article (https://doi.org/10.1038/s41379-018-0056-0) contains supplementary material, which is available to authorized users.

\footnotetext{
Chunyan Wu

Wuchunyan581@163.com

$\triangle$ Chang Chen

chenthoracic@163.com

1 Department of Thoracic Surgery, Shanghai Pulmonary Hospital, Tongji University School of Medicine, Shanghai, People's Republic of China
}

\section{Introduction}

The International Association for the Study of Lung Cancer, the American Thoracic Society and the European Respiratory Society classification for lung adenocarcinoma introduced two new terms: "adenocarcinoma in situ" and "minimally invasive adenocarcinoma". Adenocarcinoma in situ and minimally invasive adenocarcinoma was defined as adenocarcinoma smaller than $3 \mathrm{~cm}$ with a pure lepidic

2 Department of Pathology, Shanghai Pulmonary Hospital, Tongji University School of Medicine, Shanghai, People's Republic of China

3 Tongji University School of Medicine, Shanghai, People's Republic of China 
pattern and lepidic predominant pattern with invasive component $\leq 5 \mathrm{~mm}$, respectively. Disease-free survival and overall survival was almost $100 \%$ after complete resection $[1,2]$.

Further, sublobar resection was demonstrated to be sufficient for adenocarcinoma in situ and minimally invasive adenocarcinoma [3]. To choose patients for sublobar resection based on types of adenocarcinoma, requires a precise preoperative and intraoperative diagnosis. Thoracic surgeons utilize three methods: CT scan, preoperative biopsy and intraoperative frozen section. Many studies have been done to identify the histology based on the evidence of radiological features [4-6]. However, it's still difficult to apply these models to clinical use because of their unsatisfactory accuracy. The peripheral location of tumor limits the use of transbronchial biopsy and transthoracic fineneedle biopsy due to difficulties in localization and sampling.

Frozen sectiontion was a potentially effective method to identify the types of adenocarcinoma. However, the number of studies about the accuracy of frozen section is relatively small. Marchevsky reported 95\% cases were accurately classified as neoplastics or nonneoplastic lesions. Specificity was $100 \%$ [7]. The overall concordance rate between frozen section and final pathology surpassed $80 \%$ when diagnosing each type of adenocarcinoma defined by the new classification. Frozen section error mainly occurred in diagnosing adenocarcinoma in situ and minimally invasive adenocarcinoma. The concordance rate was only $63 \%[3,8$, 9]. As such, our study focused on evaluating the diagnostic performance of frozen section for the new classification of adenocarcinoma, in particular for adenocarcinoma in situ and minimally invasive adenocarcinoma and aimed to discover predictive factors for invasive adenocarcinoma as the final diagnosis in cases intraoperatively diagnosed as adenocarcinoma in situ and minimally invasive adenocarcinoma.

\section{Materials and methods}

\section{Patient selection}

All pathological reports of pulmonary nodules in 2015 and 2016 from Shanghai Pulmonary Hospital were collected. Inclusion criteria were a follows: (1) Atypical adenomatous hyperplasia, adenocarcinoma in situ, minimally invasive adenocarcinoma or invasive adenocarcinoma. (2) Primary tumor verified by immunohistochemistry and medical history. (3) Frozen section diagnosis was clear without deferral. We included 1111 adenocarcinomas from January to March 2016. Four-hundred and thirty-six cases intraoperatively diagnosed as adenocarcinoma in situ or minimally invasive adenocarcinoma in the same period were included in a derivation cohort. Five-hundred and seven cases from April to June 2016 and 379 cases from January to March 2015 were included in validation cohort 1 and 2 , respectively.

\section{Data collection}

Tumor size was measured in fresh specimen, and pathologic reports were collected for all adenocarcinomas. The clinicopathologic data, including age, sex, tumor size, subpleural nodule or not, location of tumor, carcinoembryonic antigen (ug/L), smoking status, frozen section results, final pathology results, consolidation/tumor ratio and operation mode of derivation and validation cohorts was retrieved. A tumor with no distance from tumor margin to visceral pleura in a CT scan was defined as a subpleural nodule. Invasive adenocarcinoma subtypes, lymph node metastasis and vessel invasion were reviewed for invasive adenocarcinoma in the three cohorts.

\section{Intraoperative frozen section diagnosis}

Tumors were diagnosed by pathologists immediately after being removed by a thoracic surgeon. The specimen was sliced at the largest diameter to one block for sampling. Two or three levels of tissue section were taken for diagnosis. Most frozen section results were reported by two senior pathologists with an agreement. When there was a disagreement, a third senior pathologist was invited to diagnose the case. Results were reported according to International Association for the Study of Lung Cancer/American Thoracic Society/European Respiratory Society classification of lung adenocarcinoma.

\section{Determination the reasons for frozen section underestimation of invasive adenocarcinoma}

There were three causes: Sampling error, interpretation error and suboptimal frozen section quality. To determine the reason, invasive adenocarcinomas in the three cohorts were retrospectively reviewed by six senior pathologists $(\mathrm{CW}$, HX, LZ, YH, ZD, JG) and consensus was reached after the discussion. First, the quality of frozen section was subjectively evaluated as optimal or suboptimal. A frozen section with optimal quality should reach the following criteria: (1) integrate tissue section; (2) clear stain and cell borderline; (3) clear comparison between nucleus and plasma; (4) no cutting marks, ice crystal and other abnormal tissue. If the poor quality influenced the diagnosis, the misdiagnosis was attributed to suboptimal quality. Slides with optimal quality went to the next step. Second, If the 
diagnosis of frozen section slides was changed to invasive adenocarcinoma after review, the misdiagnosis was attributed to interpretation error. If frozen section results were still adenocarcinoma in situ or minimally invasive adenocarcinoma after review, the misdiagnosis was attributed to sampling error. Third, the following two kinds of sampling errors were analyzed: (1) sampling insufficiency from the frozen tissue; (2) sampling insufficiency from non-frozen tissue. If the invasive size of levels from frozen tissue was $>5 \mathrm{~mm}$ (no matter how large the invasive size of levels from non-frozen tissue was), the misdiagnosis was attributed to sampling insufficiency from the frozen tissue. If the invasive size of levels of blocks made from non-frozen tissue was $>5 \mathrm{~mm}$ and the invasive size of levels of blocks made from frozen tissue was $<5 \mathrm{~mm}$, the misdiagnosis was attributed to sampling insufficiency from the non-frozen tissue.

\section{Statistical analysis}

We used $\chi^{2}$ test or Fisher's exact test to compare the categorical variable. Logistic regression was used to find predictive factors. Histograms were used to present the distribution of positive results. Statistical significance was set at 0.05. Statistical analysis was performed utilizing SPSS version 19.0 (SPSS, Chicago, IL).

\section{Results}

\section{Overall diagnostic accuracy and association with tumor size}

Frozen section and final pathology results of 1111 adenocarcinomas are shown in Table 1 . There were 91 cases with discordant frozen section and final pathology results. The overall concordance rate was $92 \%$. All atypical adenomatous hyperplasia were precisely diagnosed, while only $74 \%$ minimally invasive adenocarcinoma got correct diagnosis. Frozen section errors were underestimation, except for 1 adenocarcinoma in situ and 8 minimally invasive adenocarcinoma diagnosed as invasive adenocarcinoma by frozen section.

Diagnostic performance is shown in Table 2. Accuracy, sensitivity and specificity for most types surpassed $90 \%$. These results demonstrated that frozen section was a precise intraoperative diagnostic method for adenocarcinoma. However, the sensitivity for diagnosing minimally invasive adenocarcinoma $(74 \%)$ was significantly lower than other performance data.

All cases were separated to two groups by tumor size (Table 3). The concordance rate between frozen section and final pathology in tumors $\leq 1 \mathrm{~cm}$ was lower than that in tumors $>1 \mathrm{~cm}(87$ vs. $96 \%, P<0.01)$. This was also the case
Table1 Diagnostic accuracy of frozen section

\begin{tabular}{|c|c|c|c|c|c|c|c|c|c|}
\hline \multirow[t]{3}{*}{ Frozen section } & \multicolumn{8}{|c|}{ Final pathology } & \multirow{3}{*}{$\begin{array}{l}\text { Total (1111) } \\
\text { No. }\end{array}$} \\
\hline & \multicolumn{2}{|c|}{$\begin{array}{l}\text { Atypical } \\
\text { adenoma- } \\
\text { tous } \\
\text { hyperpla- } \\
\text { sia (39) }\end{array}$} & \multicolumn{2}{|c|}{$\begin{array}{l}\text { Adeno- } \\
\text { carci- } \\
\text { noma } \\
\text { in situ } \\
(271)\end{array}$} & \multicolumn{2}{|c|}{$\begin{array}{l}\text { Mini- } \\
\text { mally } \\
\text { invasive } \\
\text { adenocar- } \\
\text { cinoma } \\
(166)\end{array}$} & \multicolumn{2}{|c|}{$\begin{array}{l}\text { Invasive } \\
\text { adenocar- } \\
\text { cinoma } \\
(635)\end{array}$} & \\
\hline & No. & $\%$ & No. & $\%$ & No. & $\%$ & No. & $\%$ & \\
\hline Atypical adenomatous hyperplasia & 39 & 100 & 16 & 6 & 0 & 0 & 0 & 0 & 55 \\
\hline Adenocarcinoma in situ & 0 & 0 & 251 & 93 & 34 & 20 & 5 & 0 & 290 \\
\hline Minimally invasive adenocarcinoma & 0 & 0 & 0 & 0 & 123 & 74 & 23 & 4 & 146 \\
\hline Invasive adenocarcinoma & 0 & 0 & 1 & 0 & 8 & 5 & 607 & 96 & 616 \\
\hline Benign & 0 & 0 & 3 & 1 & 1 & 1 & 0 & 0 & 4 \\
\hline
\end{tabular}

\begin{tabular}{llllll}
\hline Category & Accuracy\% & Sensitivity\% & Specificity\% & PPV\% & NPV\% \\
\hline Atypical adenomatous hyperplasia & 99 & 100 & 99 & 71 & 100 \\
Adenocarcinoma in situ & 95 & 93 & 95 & 87 & 98 \\
Minimally invasive adenocarcinoma & 94 & 74 & 98 & 84 & 96 \\
Invasive adenocarcinoma & 97 & 96 & 98 & 99 & 94 \\
\hline
\end{tabular}

All values shown as percent

$P P V$ positive predictive value, $N P V$ negative predictive value
Table 2 Diagnostic performance of frozen section 
Table 3 Concordance rate between frozen section and final pathology in each type stratified by tumor size

\begin{tabular}{llll}
\hline Final pathology & \multicolumn{2}{l}{ Tumor size } & \\
\cline { 2 - 3 } & $\leq 1 \mathrm{~cm}$ & $>1 \mathrm{~cm}$ & $P$ value \\
\hline Overall & $87 \%$ & $96 \%$ & $<0.01$ \\
Atypical adenomatous hyperplasia & $100 \%$ & $100 \%$ & $>0.99$ \\
Adenocarcinoma in situ & $92 \%$ & $100 \%$ & 0.61 \\
Minimally invasive adenocarcinoma & $75 \%$ & $72 \%$ & 0.73 \\
Invasive adenocarcinoma & $84 \%$ & $97 \%$ & $<0.01$ \\
\hline
\end{tabular}

between invasive adenocarcinoma $\leq 1 \mathrm{~cm}$ and invasive adenocarcinoma $>1 \mathrm{~cm}$ (84 vs. $97 \%, P<0.01)$. All atypical adenomatous hyperplasia were correctly diagnosed. There was no statistical difference between the concordance rate for adenocarcinoma in situ $\leq 1 \mathrm{~cm}$ and $>1 \mathrm{~cm}$ (92 vs. $100 \%$, $P=0.61$ ). This was the same between minimally invasive adenocarcinoma $\leq 1 \mathrm{~cm}$ and $>1 \mathrm{~cm}$ (75 vs. $72 \%, P=0.73)$.

\section{Clinicopathologic features of the derivation and validation cohorts}

The clinicopathologic features of the derivation and validation cohorts are shown in Table 4 and Supplementary Table 1 and 2. The incidence of invasive adenocarcinoma as the final diagnosis was significant higher in minimally invasive adenocarcinoma than that in adenocarcinoma in situ in three cohorts.

\section{Predictive factors for invasive adenocarcinoma as the final diagnosis in the derivation cohort}

Final pathology proved the diagnosis of $28(6 \%)$ invasive adenocarcinomas. Univariate analysis showed age $(P=$ $0.002)$, sex $(P=0.033)$, smoking status $(P=0.001)$, tumor size $(P<0.001)$, subpleural nodule $(P=0.07)$ were associated with a final diagnosis of invasive adenocarcinoma. Multivariate analysis showed that tumor size was the only independent predictive factors $(<1 \mathrm{~cm}$ : reference; $1-14 \mathrm{~cm}$ : odds ratio, 5.678; $>1.5 \mathrm{~cm}$ : odds ratio: 5.878; $P=0.001$ ). (Table 5) The distribution of invasive adenocarcinoma by tumor size is shown in Fig. 1. Seven $(7 / 301,2 \%)$ invasive adenocarcinomas were found in tumors $<1 \mathrm{~cm}$, one $(1 / 234)$ from adenocarcinoma in situ and $6(6 / 67,9 \%)$ from minimally invasive adenocarcinoma. Fifteen $(15 / 101,15 \%)$ cases were found in tumors with a size from 1 to $1.4 \mathrm{~cm}$, four $(4 / 44,9 \%)$ from adenocarcinoma in situ and $11(11 / 57$, $19 \%)$ from minimally invasive adenocarcinoma. Six (6/34, $18 \%$ ) invasive adenocarcinomas were found in tumors $\geq 1.5$ $\mathrm{cm}$, all cases $(6 / 22,27 \%)$ from minimally invasive adenocarcinoma. Twenty-eight misdiagnosed invasive adenocarcinomas consisted of 26 (92\%) lepidic predominant type,
$1(4 \%)$ papillary and $1(4 \%)$ acinar predominant type. There were no positive $\mathrm{N} 1, \mathrm{~N} 2$ node, pleural, lymphatic and vascular invasion cases found.

\section{Predictive factors for invasive adenocarcinoma to be the final diagnosis in validation cohorts}

Risk factors derived by univariate analysis above were not all confirmed in validation cohorts. Age $(P=0.006)$, consolidation/tumor ratio $(P=0.002)$ and tumor size $(P<0.001)$ were risk factors in validation cohort 1 . Consolidation/tumor ratio $(P=0.002)$, tumor size $(P<0.001)$ were risk factors in validation cohort 2 . However, multivariate analysis still proved that tumor size was the only risk factor (Validation cohort 1: $1-1.4 \mathrm{~cm}$ : odds ratio, $21.000 ;>1.5 \mathrm{~cm}$ : odds ratio, 49.984; $P=0.003$. Validation cohort $2: 1-1.4 \mathrm{~cm}$ : odds ratio: $18.855 ;>1.5 \mathrm{~cm}$ : odds ratio, 107.330; $P<0.001$ ) (Supplementary Table 3 and 4). The distribution of invasive adenocarcinoma based on tumor size is shown in Supplementary Figure 1 and 2 . In validation cohort 1 , no invasive adenocarcinoma was found in 362 tumors $<1 \mathrm{~cm}$. Nine $(7 \%)$ invasive adenocarcinomas were found in 121 tumors with a size from 1 to $1.5 \mathrm{~cm}$. Five (21\%) invasive adenocarcinomas were found in 24 tumors $\geq 1.5 \mathrm{~cm}$. Eleven $(79 \%)$ cases were lepidic predominant and three $(21 \%)$ were acinar predominant. In validation cohort 2 , one invasive adenocarcinoma was found in 270 tumors $<1 \mathrm{~cm}$. Seven (9\%) invasive adenocarcinomas were found in 81 tumors with a size from 1 to $1.4 \mathrm{~cm}$. Nine (32\%) invasive adenocarcinomas were found in 28 tumors $\geq 1.5 \mathrm{~cm}$. Seventeen $(100 \%)$ cases were lepidic predominant. There were no positive N1, N2 node, pleural, lymphatic and vascular invasion cases found.

\section{Reasons for underestimation of invasive adenocarcinoma by frozen section}

For 59 invasive adenocarcinomas in the three cohorts, 37 (63\%) were sampling error, 17 (29\%) were interpretation error and 5 (8\%) were suboptimal quality (Fig. 2). Sampling due to leveling from frozen tissue only contributed to the 2 $(5 \%, 2 / 37)$ misdiagnosis. The invasive size was $4 \mathrm{~mm}$ in frozen section and $6 \mathrm{~mm}$ in final pathology for both cases. Thirty-five $(95 \%, 35 / 37)$ cases was attributed to the increasing of invasive size in sections from non-frozen tissue. The invasive size was $2.0 \pm 1.7 \mathrm{~mm}$ in frozen section, $8.8 \pm$ $2.7 \mathrm{~mm}$ in final pathology $(P<0.01)$. The mean invasive size increased by $6.8 \mathrm{~mm}$. As to interpretation error, the invasive size was $6.3 \pm 1.1 \mathrm{~mm}$ in frozen section, $8.5 \pm 1.6 \mathrm{~mm}$ in final pathology $(P<0.01)$. Although the misdiagnosis was caused by measurement error of invasive size in intraoperative frozen section, the mean value still increased by $2.2 \mathrm{~mm}$ comparing to revised frozen section results. Figure 3 shows the changes of invasive size in each case. 
Table 4 Clinicopathologic features of the derivation cohort

\begin{tabular}{|c|c|c|c|c|}
\hline \multirow[t]{2}{*}{ Characteristics } & \multirow[b]{2}{*}{$\begin{array}{l}\text { Total }(n= \\
436)\end{array}$} & \multicolumn{2}{|l|}{ Frozen section } & \multirow[b]{2}{*}{$P$ value } \\
\hline & & $\begin{array}{l}\text { Adenocarcinoma in situ ( } n \\
=290)\end{array}$ & $\begin{array}{l}\text { Minimally invasive } \\
\text { adenocarcinoma }(n=146)\end{array}$ & \\
\hline \multicolumn{5}{|l|}{ Age } \\
\hline$\leq 60$ & $291(67)$ & $217(75)$ & $74(51)$ & \multirow[t]{2}{*}{$<0.001$} \\
\hline$>60$ & $145(33)$ & $73(25)$ & $72(49)$ & \\
\hline \multicolumn{5}{|l|}{ Sex } \\
\hline Male & $137(31)$ & $84(29)$ & $53(36)$ & \multirow[t]{2}{*}{0.127} \\
\hline Female & $299(69)$ & $206(71)$ & $93(64)$ & \\
\hline \multicolumn{5}{|l|}{ Smoking } \\
\hline Yes & $31(7)$ & $18(6)$ & $13(9)$ & \multirow[t]{2}{*}{0.301} \\
\hline No & $405(93)$ & $272(94)$ & $133(91)$ & \\
\hline \multicolumn{5}{|l|}{ Tumor size $(\mathrm{cm})$} \\
\hline$<1$ & $301(69)$ & $234(81)$ & $67(46)$ & \multirow[t]{3}{*}{$<0.001$} \\
\hline $1-1.4$ & $101(23)$ & $44(15)$ & $57(39)$ & \\
\hline$\geq 1.5$ & $34(8)$ & $12(4)$ & $22(15)$ & \\
\hline \multicolumn{5}{|l|}{ Subpleural } \\
\hline Yes & $108(25)$ & $69(24)$ & 39 (27) & \multirow[t]{2}{*}{0.505} \\
\hline No & $328(75)$ & $221(76)$ & $107(73)$ & \\
\hline \multicolumn{5}{|c|}{ Consolidation/Tumor ratio } \\
\hline$>0.25$ & $98(22)$ & $50(17)$ & $48(33)$ & \multirow[t]{2}{*}{$<0.001$} \\
\hline$\leq 0.25$ & $338(78)$ & $240(83)$ & $98(67)$ & \\
\hline \multicolumn{5}{|l|}{ Location } \\
\hline Upper & $281(64)$ & $183(63)$ & $98(67)$ & \multirow[t]{3}{*}{0.008} \\
\hline Middle & $37(9)$ & $18(6)$ & $19(13)$ & \\
\hline Lower & $118(27)$ & $89(31)$ & $29(20)$ & \\
\hline \multicolumn{5}{|c|}{ Carcinoembryonic antigen $(\mu \mathrm{g} / \mathrm{L})$} \\
\hline$\geq 5$ & $27(6)$ & $5(2)$ & $22(15)$ & \multirow[t]{2}{*}{$<0.001$} \\
\hline$<5$ & 409 (94) & $285(98)$ & $124(85)$ & \\
\hline \multicolumn{5}{|l|}{ Final pathology } \\
\hline Others & $408(94)$ & $285(98)$ & $123(84)$ & \multirow[t]{2}{*}{$<0.001$} \\
\hline IA & $28(6)$ & $5(2)$ & $23(16)$ & \\
\hline \multicolumn{5}{|l|}{ Surgical type } \\
\hline Lobectomy & $101(23)$ & $54(19)$ & $47(32)$ & \multirow[t]{2}{*}{0.003} \\
\hline Limited resection & $335(77)$ & $236(81)$ & $99(68)$ & \\
\hline
\end{tabular}

Values are presented as $n(\%)$

\section{Impact of interobserver variability for frozen section}

In our clinical practice, six pathologists in total diagnosed 1322 frozen section in three cohorts. Most frozen section results were reported by 2 senior pathologists with an agreement. When there was a disagreement, a third senior pathologist was invited to diagnose the case. Among 1322 cases, first diagnosis of $198(15 \%, 198 / 1322)$ frozen sections were different between the two pathologists. Onehundred and fifty-five $(78 \%, 155 / 198)$ cases got diagnosed correctly after a third pathologist invited. Forty-three (22\%, 43/198) cases did not get correct intraoperative diagnosis. However, the postoperative changing of diagnosis was limited to the range among atypical adenomatous hyperplasia, adenocarcinoma in situ and minimally invasive adenocarcinoma in 39 cases. Four cases $(2 \%, 4 / 198)$ upstaged to the invasive adenocarcinomas were attributed to sampling error after review. This part of cases only took $0.3 \%$ in three cohorts.

\section{Clinical impact of frozen section underestimation}

Limited resection rate was 77\% (335/436), 76\% (384/507) and $70 \%(266 / 379)$ in the derivation cohort, validation cohort 1 and 2, respectively. For 59 invasive adenocarcinomas in the three cohorts, twenty-six $(44 \%, 26 / 59)$ patients 
Table 5 Univariate and multivariate analysis of derivation cohort

\begin{tabular}{|c|c|c|c|c|}
\hline \multirow[t]{2}{*}{ Predictors } & \multicolumn{2}{|l|}{ Univariate } & \multicolumn{2}{|l|}{ Multivariate } \\
\hline & Odds ratio $(95 \% \mathrm{CI})$ & $P$ value & Odds ratio $(95 \% \mathrm{CI})$ & $P$ value \\
\hline Age $(>60 / \leq 60)$ & $3.4(1.5-7.4)$ & 0.002 & $2.32(1.0-5.5)$ & 0.055 \\
\hline Sex (Female/Male) & $0.4(0.2-0.9)$ & 0.033 & $0.59(0.2-1.6)$ & 0.304 \\
\hline Smoking (Yes/No) & $5.3(2.1-13.8)$ & 0.001 & $3.25(0.9-11.2)$ & 0.06 \\
\hline \multicolumn{5}{|l|}{ Tumor size $(\mathrm{cm})$} \\
\hline$<1$ & reference & & reference & \\
\hline $1-1.4$ & $7.3(2.9-18.5)$ & $<0.001$ & $5.77(2.2-15.2)$ & $<0.001$ \\
\hline$\geq 1.5$ & $9.0(2.8-28.6)$ & $<0.001$ & $5.88(1.7-19.8)$ & 0.004 \\
\hline Subpleural (Yes/No) & $2.1(0.9-4.6)$ & 0.07 & $1.66(0.7-3.9)$ & 0.244 \\
\hline Consolidation/tumor ratio $(>0.25 / \leq 0.25)$ & $1.4(0.6-3.2)$ & 0.491 & & \\
\hline \multicolumn{5}{|l|}{ Location } \\
\hline Upper & reference & 0.533 & & \\
\hline Middle & $1.9(0.6-5.9)$ & 0.280 & & \\
\hline Lower & $1.0(0.4-2.4)$ & 0.964 & & \\
\hline Carcinoembryonic antigen $(\mu \mathrm{g} / \mathrm{L})(\geq 5 /<5)$ & $1.8(0.2-15.3)$ & 0.575 & & \\
\hline
\end{tabular}

CI confidential interval a

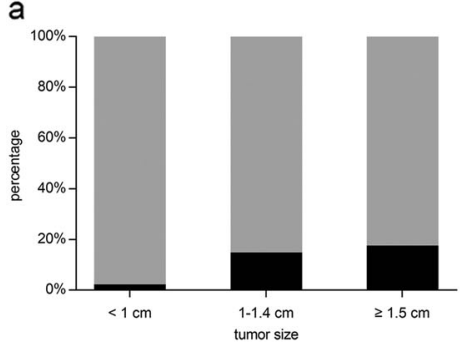

b

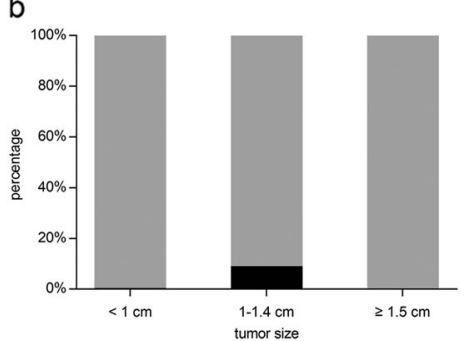

c

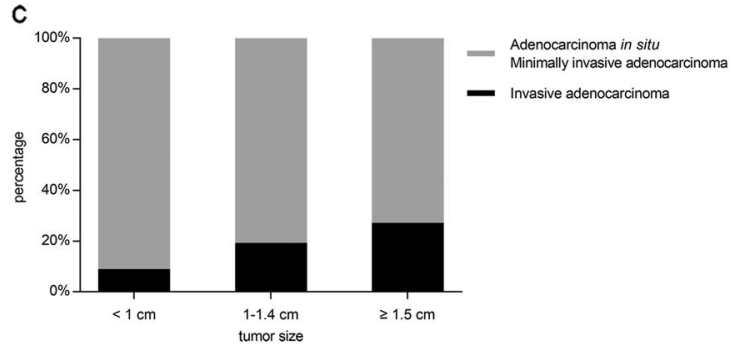

Fig. 1 Histograms showing proportion of invasive adenocarcinoma in the derivation cohort. a Entire cohort. b Adenocarcinoma in situ by frozen section. c Minimally invasive adenocarcinoma by frozen section

underwent limited resection. Among them, $8(31 \%, 8 / 26)$ were compromised surgery because of cardiopulmonary function $(13 \%, 1 / 8)$, multiple lesions $(74 \%, 6 / 8)$ or age $>75$ $(13 \%, 1 / 8)$. Final reports were all lepidic predominant adenocarcinoma. The remaining 18 patients were intentionally selected to undergo limited resection based on frozen section results. One patient with acinar predominant invasive adenocarcinoma and a tumor size of $1.8 \mathrm{~cm}$ underwent segmentectomy. This patient underwent a second surgery for complementary lobectomy and systematic lymph node dissection 3 month after first surgery. Another patient with papillary predominant subtype containing $20 \%$ micropapillary component and a tumor size of $0.6 \mathrm{~cm}$ underwent wedge resection. This patient denied additional surgery and was transferred to the department of medical oncology for adjuvant chemotherapy. The remaining 16 patients were all lepidic predominant invasive adenocarcinoma without solid or micropapillary component. These patients underwent no additional treatment. Thirty-three $(56 \%, 33 / 59)$ still underwent lobectomy or wedge resection plus complementary lobectomy although frozen section showed adenocarcinoma in situ or minimally invasive adenocarcinoma. The reasons for lobectomy instead of limited resection were large tumor size, "malignant" CT imaging (Consolidation/Tumor ratio $>0.5$, irregular shape, unclear borderline, pleural indentation and so on) and insufficient margin distance (e.g., tumor located closed to the hilar structure). All patients were followed up until 04 December 2017. Fortunately, there was no recurrence and death.

\section{Discussion}

The accuracy of frozen section is affected by many factors. Frozen sections of 224 consecutive primary pulmonary adenocarcinomas were reviewed by Walts et al. Tumor size, consultation by more than one pathologist and more than one sample for frozen section were associated with frozen section error and deferral. The root causes were sampling 
Fig. 2 Example of sampling error and suboptimal frozen section. a No invasive component is found in the frozen section slide, hematoxylin and eosin (H\&E), $\times 100$. b A large size of invasion is found in the paraffinembedded section, H\&E, $\times 100$. c Unclear staining of cell structure in frozen section slides, $\mathrm{H} \& \mathrm{E}, \times 100$. d Invasive component was found in paraffin-embedded section, $\mathrm{H} \& \mathrm{E}, \times 100$
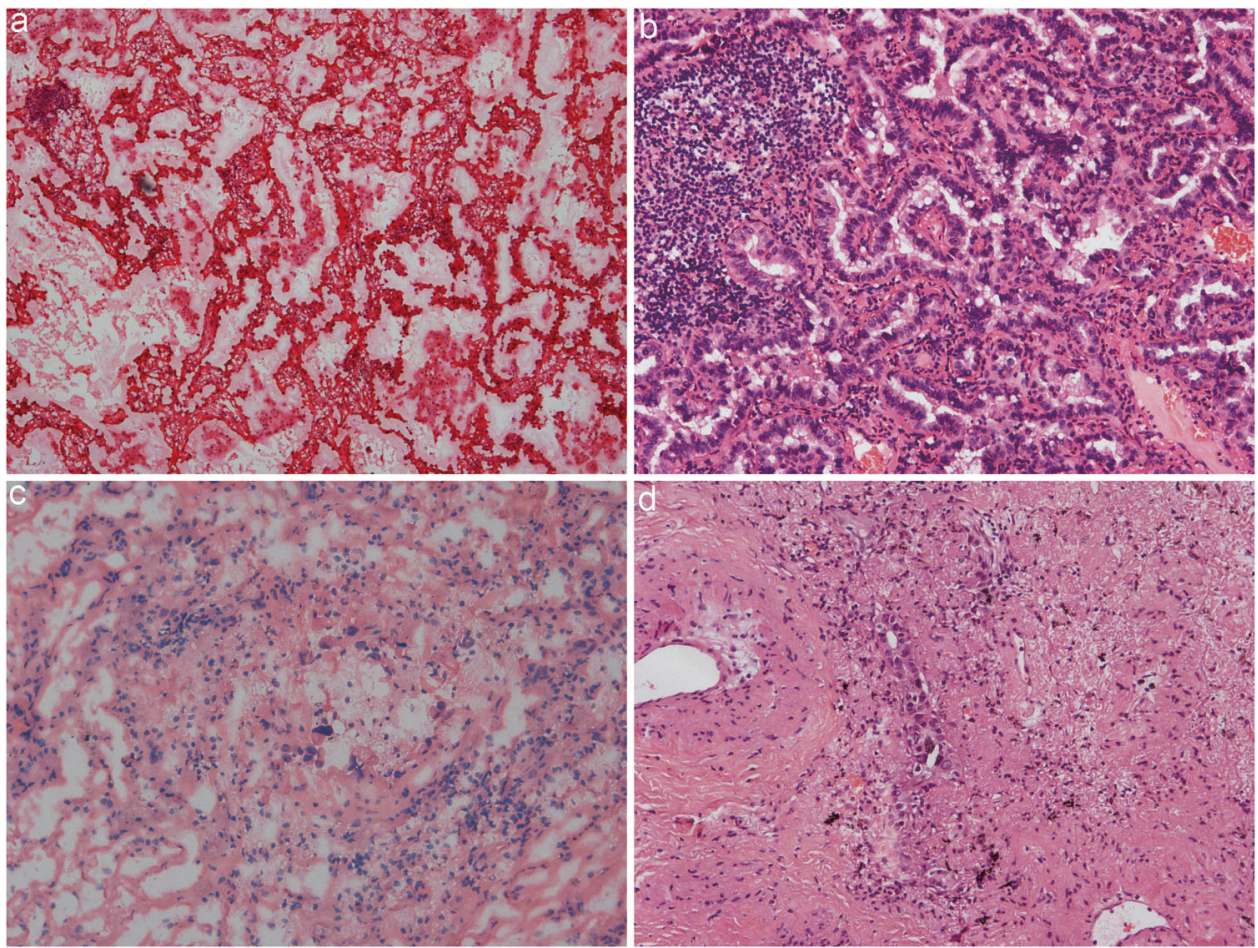

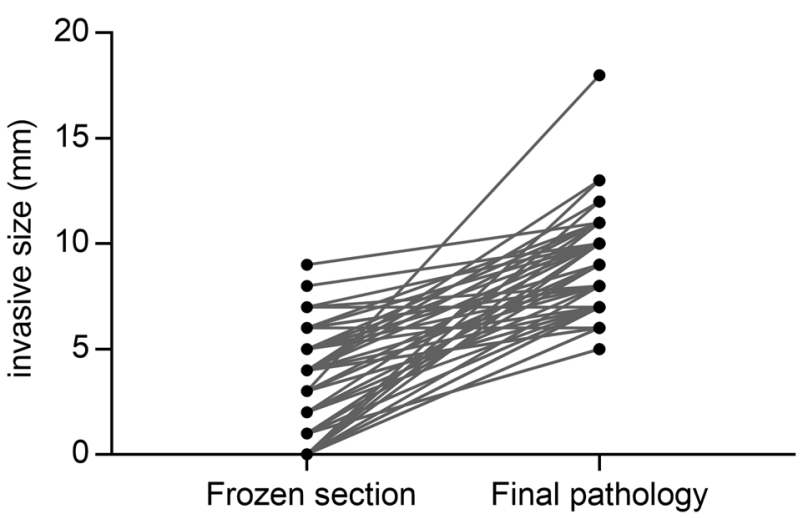

Fig. 3 Changes of invasive size from frozen section to paraffinembedded section in the derivation and validation cohorts

error, interpretation error and suboptimal frozen section quality [8]. Most studies found frozen section was more accurate in diagnosing larger tumors. Marchevsky et al. reported the diagnostic performance of frozen section in differentiating malignancy from benign lesion smaller than $1.5 \mathrm{~cm}$. Specificity was $100 \%$. Sensitivity was higher for tumors $\geq 1.1 \mathrm{~cm}$ (94 vs. 87\%) [7]. For the new classification of adenocarcinoma, the rate of frozen section error and deferral was twice higher in tumor $<1 \mathrm{~cm}$ (32 vs. 17\%) [8]. The error rate decreased in the later study due to a longer practice period for pathologists $(\leq 1 \mathrm{~cm}, 20.4 \% ;>1 \mathrm{~cm}, 9 \%)$ [3]. This effect may be caused by the difficulties of processing tumors smaller than $1 \mathrm{~cm}$ and the accuracy of one slice of a small tumor could be easily affected by a heterogeneous character. Our study also obtained similar results in adenocarcinoma. However, this was not validated in atypical adenomatous hyperplasia, adenocarcinoma in situ and minimally invasive adenocarcinoma. Other studies did not show the data of concordance rate according to final pathology results and tumor size to support our findings. This may need further investigation.

The diagnostic accuracy of frozen section was different among atypical adenomatous hyperplasia, adenocarcinoma in situ, minimally invasive adenocarcinoma and invasive adenocarcinoma. According to Walts' study, 97\% cases of invasive adenocarcinomas were correctly diagnosed. Only $59 \%$ adenocarcinoma in situ and $46 \%$ minimally invasive adenocarcinoma obtained concordant intraoperative result [8]. In a recent study, the concordance rate of frozen section diagnosis in 136 cases of adenocarcinoma in situ and minimally invasive adenocarcinoma was 63\% [9]. These studies did not obtain more accurate diagnosis because of the relatively smaller number of cases. Liu reported the concordance rate of adenocarcinoma in situ, minimally invasive adenocarcinoma and invasive adenocarcinoma was $74 \%, 76 \%$ and $93 \%$, respectively. Most discrepant cases were underestimation of adenocarcinoma in situ and minimally invasive adenocarcinoma. There were 100 discrepancies in adenocarcinoma in situ and minimally invasive adenocarcinoma, which took $80 \%$ of all frozen section errors [3]. In our results, concordance rate in adenocarcinoma in situ $(93 \%)$ was significantly higher than the 
rate of other studies. The rate in minimally invasive adenocarcinoma and invasive adenocarcinoma was 74 and $96 \%$, respectively, which is similar to Liu's report.

Why minimally invasive adenocarcinomas are difficult to diagnose? Before the new classification, the main concern for pathologists was the presence of invasion. The diagnosis of minimally invasive adenocarcinoma according to new classification of International Association for the Study of Lung Cancer/American Thoracic Society/European Respiratory Society focuses on the size of invasion. Measurement of invasive size is easily influenced by a suboptimal frozen section, presence of fibrosis or scar, collapsed alveolar spaces and subjective factors. To improve accuracy, it's necessary to invent new methods or technology.

Invasive adenocarcinoma misdiagnosed as adenocarcinoma in situ and minimally invasive adenocarcinoma is likely to undergo potentially insufficient resection. As such, we wanted to find some predictive factors for invasive adenocarcinoma as the final diagnosis among intraoperatively diagnosed adenocarcinoma in situ and minimally invasive adenocarcinoma cases. Tumor size measured in fresh specimen, consolidation/tumor ratio, carcinoembryonic antigen value and subpleural nodule were included in our predictive model. Tumor size is associated with invasiveness and the infiltration range of larger tumors may exceed that observed in frozen section because of sampling error [9]. Radiological features of ground-glass opacity are commonly used to evaluate invasiveness. The radiological criteria of noninvasive adenocarcinoma according to consolidation/tumor ratio has been used to clinical trial JCOG0802 and JCOG0804 [10, 11]. Subpleural nodules are likely to be misdiagnosed because frozen section has difficulties in identifying pleural invasion. Basic characters like age, sex, smoking status and tumor location were also included. Our results showed that tumor size was the only predictive factor in multivariate logistic analysis. At the background of known frozen section results, other factors lost their predictive value of invasiveness because of the high accuracy of frozen section diagnosis. Therefore, surgeons could predict the final result according to the frozen section result and tumor size. Sampling error was the leading cause for this specific error. For large tumors, increasing blocks for frozen section sampling may decrease the error rate and prospective study is needed.

For pulmonary nodule smaller than $2 \mathrm{~cm}$, lobectomy or sublobar resection is still a controversial issue. A randomized trial conducted by Lung Cancer Study Group in 1995 recommended lobectomy as the standard procedure for T1N0M0 patients [12]. In recent years, some retrospective studies revealed no difference in survival between lobectomy and sublobar resection [13, 14], while some still showed worse prognosis for sublobar resection [15-17].
However, most studies did not take the new classification of adenocarcinoma into account. Limited resection was not associated with a worse prognosis in patients in adenocarcinoma in situ and minimally invasive adenocarcinoma. Liu et al. suggested that thoracic surgeons could select patients intraoperatively diagnosed as adenocarcinoma in situ and minimally invasive adenocarcinoma for limited resection [3]. However, our study showed that tumors $\geq 1 \mathrm{~cm}$ intraoperatively diagnosed as adenocarcinoma in situ and minimally invasive adenocarcinoma were more likely to be invasive adenocarcinoma proved by final pathology, in particular, for cases intraoperatively diagnosed as minimally invasive adenocarcinoma. The incidence of insufficient diagnosis was nearly $20 \%$ in tumors $\geq 1.5 \mathrm{~cm}$. In the derivation cohort, 28 misdiagnosed invasive adenocarcinoma consisted of $26(92 \%)$ lepidic predominant type, $1(4 \%)$ papillary and $1(4 \%)$ acinar predominant type. There were no positive N1, N2 node, lymphatic and vascular invasion found. Although most misdiagnosed cases were lepidic predominant and limited resection seemed to be sufficient reported by retrospective studies [18, 19]. This finding has not been validated by clinical trials yet and the extent of lymph node dissection remains controversial.

In conclusion, frozen section is a precise intraoperative diagnostic method. For thoracic surgeons, frozen section results and tumor size measured in fresh specimens should be considered jointly to predict the final diagnosis. For pathologists, frozen section diagnosis of adenocarcinoma in situ and minimally invasive adenocarcinoma should be considered cautiously for tumor $\geq 1 \mathrm{~cm}$ to avoid insufficient resection. The cost effectiveness of more blocks for frozen section sampling needs to be evaluated by prospective studies.

Acknowledgements This study was supported by the projects from Shanghai Hospital Development Center (SHDC12015116), Health and Family Planning Commission of Shanghai Municipality (20154Y0097) and Shanghai Pujiang Program (15PJD034).

\section{Compliance with ethical standards}

Conflict of interest The authors declare that they have no conflict of interest.

\section{References}

1. Travis WD, Brambilla E, Noguchi M, et al. International association for the study of lung cancer/american thoracic society/ european respiratory society international multidisciplinary classification of lung adenocarcinoma. J Thorac Oncol. 2011;6:244-85.

2. Yoshizawa A, Motoi N, Riely GJ, et al. Impact of proposed IASLC/ATS/ERS classification of lung adenocarcinoma: prognostic subgroups and implications for further revision of staging based on analysis of 514 stage I cases. Mod Pathol. 2011;24:653-64. 
3. Liu S, Wang R, Zhang Y, et al. Precise diagnosis of intraoperative frozen section is an effective method to guide resection strategy for peripheral small-sized lung adenocarcinoma. J Clin Oncol. 2016;34:307-13.

4. Nakamura H, Saji H, Shinmyo T, et al. Close association of IASLC/ ATS/ERS lung adenocarcinoma subtypes with glucose-uptake in positron emission tomography. Lung Cancer. 2015;87:28-33.

5. Lim HJ, Ahn S, Lee KS, et al. Persistent pure ground-glass opacity lung nodules $/=10 \mathrm{~mm}$ in diameter at CT scan: histopathologic comparisons and prognostic implications. Chest. 2013;144:1291-9.

6. Chae HD, Park CM, Park SJ, et al. Computerized texture analysis of persistent part-solid ground-glass nodules: differentiation of preinvasive lesions from invasive pulmonary adenocarcinomas. Radiology. 2014;273:285-93.

7. Marchevsky AM, Changsri C, Gupta I, et al. Frozen section diagnoses of small pulmonary nodules: accuracy and clinical implications. Ann Thorac Surg. 2004;78:1755-9.

8. Walts AE, Marchevsky AM. Root cause analysis of problems in the frozen section diagnosis of in situ, minimally invasive, and invasive adenocarcinoma of the lung. Arch Pathol Lab Med. 2012;136:1515-21.

9. He P, Yao G, Guan Y, et al. Diagnosis of lung adenocarcinoma in situ and minimally invasive adenocarcinoma from intraoperative frozen sections: an analysis of 136 cases. J Clin Pathol. 2016;69:1076-80.

10. Suzuki K, Koike T, Asakawa T, et al. A prospective radiological study of thin-section computed tomography to predict pathological noninvasiveness in peripheral clinical IA lung cancer (Japan Clinical Oncology Group 0201). J Thorac Oncol. 2011;6:751-6.
11. Lee KH, Goo JM, Park SJ, et al. Correlation between the size of the solid component on thin-section CT and the invasive component on pathology in small lung adenocarcinomas manifesting as ground-glass nodules. J Thorac Oncol. 2014;9:74-82.

12. Ginsberg RJ,Rubinstein LV, Randomized trial of lobectomy versus limited resection for T1 N0 non-small cell lung cancer. Lung Cancer Study Group. Ann Thorac Surg. 1995;60:615-22.

13. Wisnivesky JP, Henschke CI, Swanson S, et al. Limited resection for the treatment of patients with stage IA lung cancer. Ann Surg. 2010;251:550-4.

14. Zhong C, Fang W, Mao T, et al. Comparison of thoracoscopic segmentectomy and thoracoscopic lobectomy for small-sized stage IA lung cancer. Ann Thorac Surg. 2012;94:362-7.

15. Stefani A, Nesci J, Casali C, et al. Wedge resection versus lobectomy for T1N0 non-small cell lung cancer. Minerva Chir. 2012;67:489-98.

16. Wolf AS,Richards WG,Jaklitsch MT, et al. Lobectomy versus sublobar resection for small ( $2 \mathrm{~cm}$ or less) non-small cell lung cancers. Ann Thorac Surg. 2011;92:1819-23.

17. Dai C, Shen J, Ren Y, et al. Choice of surgical procedure for patients with non-small-cell lung cancer $/=1 \mathrm{~cm}$ or 1 to $2 \mathrm{~cm}$ among lobectomy, segmentectomy, and wedge resection: a population-based study. J Clin Oncol. 2016;34:3175-82.

18. Dembitzer FR, Flores RM, Parides MK, et al. Impact of histologic subtyping on outcome in lobar vs sublobar resections for lung cancer: a pilot study. Chest. 2014;146:175-81.

19. Cox ML, Yang CJ, Speicher PJ, et al. The role of extent of surgical resection and lymph node assessment for clinical stage i pulmonary lepidic adenocarcinoma: an analysis of 1991 patients. J Thorac Oncol. 2017;12:689-96. 